\title{
EL DESARROLLO DE LA ZONA DE BAHIA MAGDALENA UN PANORAMA BIO-SOCIO-ECONOMICO EN UNA REGION EN PLENO DESARROLLO.
}

\author{
Por:

\section{Christopher Paul Mathews}

\author{
Ciencias Marinas Vol. 2 Núm. I
}

\begin{abstract}
Se presenta un resumer breve de las existencias demersales en Bahla Magdalena. Con límites mencionados se presentan cifras que permiten una aproximución de las capturas realizables de las existencias. $v$ de su valor económico. Se presenta un panorama serio económico de iśrea.
\end{abstract}

\section{ABSTRACT}

A brief review of the present knowledge of the demersal stocks of Magdalena Bay is given. Approximate values of the likely yields from these stocks are given, together with reasonable limits; the approximate values of thea stocks are given. These results are discussed in the light of the serious economic realities of the area.

El presente trabajo fue presentado como ponencia en el $V$ Congreso Nacional de Oceanografía celebrado en Guaymas, Son. en octubre de 1974.

En una serie de cuatro capítulos en esta misma publicación se presentan algunos resultados preliminares de los trabajos ejecutados en la zona de Bahía Magdalena. Uno de los resultados más importantes, de este tipo de trabajo, especialmente en una zona relativamente desconocida, resulta ser un avance en muchos detalles de la Biología y Ecología de las especies ahí encontradas. Actualmente esto es de suma importancia puesto que de muchas de las especies se conoce poco o nada, acerca de sus ciclos de vida, crecimiento, ecología y dinámica de población; cuando estos trabajos estén ya concluídos, proporcionarán. una serie de datos básicos de importancia primordial para el conocimiento de las especies que abundan en una gran parte del litoral mexicano.

Al mismo tiempo debemos subrayar repetida y terminantemente que este tipo de resultado, valioso e importante no es la primera meta de un estudio como éste.
El contrato se designó en primer lugar como proyecto para investigar el aprovechamiento posible $y$ en particular las posibilidades de cultivo de los organismos que habiton en la zona de Bahía Magdalena, $y$ en segundo lugar para determinar la importancia de la Bahía como zona de refugio y cría, tomando muy en cuenta que la zona de trabajo está ubicada en el centro de una zona de desarrollo económico intenso, con todas las consecuencias ambientales en cuanto a contaminación y destrucción de especies y de existencias que éstos puedan causar.

Para un Biólogo Pesquero, es un deber en cualquier momento proporcionar datos sobre las posibles cosechas obtenibles de una zona con existencias precisamente definidas. Siempre y cuando se presenten con las cosechas estimadas, limites de credibilidad (más bien que de confianza estadistical, para que las superioridades tengan la información necesaria para precisar las decisiones en bases racionales $y$ responsables.

Basándonos entonces, en los trabajos presentados aquí, en los datos todavia no publicados $y$ en mis observaciones de 
campo se ha preparado una relación de las cosechas posibles, con márgenes de credibilidad. Estas cifras presentan, por así decirlo, una precipitación de lo que hasta ahora conocemos y seguramente serán revisadas en un futuro próximo.

Cualquiera de estas cifras podrian errar por un factor de $\times 2-\times 3$, pero algunos errarán del lado de la subestimación y otros de la sobreestimación. Sería el valor de $800 \mathrm{Tm}$ conservador y la de 3,000 Tm quizó exagerada. Así se concluye como un valor razonable el de 1,600 Tm para la cosecha anual excluyendo el ostión.

El valor de estas capturas variará mucho según (i) el rendimiento de carne que proporcione la especie (eq. 20-22\% para las rayas, y aproximadamente $35 \%$ para $M$. ectenes, aproximadamente $45 \%$ para Squetina californiensis); (ii) el valoi de la carne obtenida: $\$ 2.50 \mathrm{MN}$. por $\mathrm{kg}$ de pescado de escama, $\$ 30.00$ M.N. para camarón azul y café, \$15.00 M.N. para el camarón de roca; (iii) la proporción de pescado de escama en general que se destina para consumo humano.

El valor de $\$ 2.50$ M.N. para el $\mathrm{kg}$ de pescado de escama es extremadamente bajo, y uno de los problemas básicos es la falta de buen mercado y de pre. cios constantes para el pescado.

Aunque fuera de la zona de Bahía Magdalena se cotizan precios mayores, éste es el precio que reciben los pescadores. Asumiendo estos precios, el valor al pescador, de la captura sostenible de $1,600 \mathrm{Tm}$ anuales sería de $1,600,000 \times$ $\$ 2.50$ igual a $\$ 4,000,000.00$ M.N. anuales. Será algo más alto si se llega a aprovechar una porción significativa de samarón. Los valores del camarón no se calcularán puesto que las capturas actualmente realizadas se desconocen, y nuestros datos sobre la biomasa de las dos especies comerciales todavía son muy escasos. Pero tomando una actitud positiva, parece probable que el valor de la pesca conercial pudiera ser de ............ $\$ 5,000,000.00$ M.N. anuales, aunque podría ser tan bajo como $\$ 2,000,000.00$ anuales o llegar con una combinación de factores extremadamente favorables a $\$ 15,000,000.00$ de pesos. Pero mi pare- cer sería éste, probablemente un valor alto, y una cifra de $\$ 4-5,000,000.00$ anuales sería una estimación razonable que correspondería a los conocimientos actuales.

La pesca del ostión de mangle podria añadir significativamente al valor de la pesca, pero nuestros conocimientos indican que si abunda el ostión en algunas zonas, en otras es algo escaso y hasta que terminen nuestros estudios preferimos excluir esta especie de la consideración.

La posibilidad del cultivo de ostión debe considerarse. Los datos disponibles nos llevan a pensar que será una zona apta para el cultivo de ostión, en un futuro breve los investigadores de la Unidad de Ciencias Marinas comenzarán a observar el crecimiento del ostión, Crassostrea gigas mediante balsas. Los mismos investigadores lograron resultados favorables en Bahia San Quintín. Los resultados hidrográficos que tenemos disponibles nos llevan a pensar que se encontraron en Bahía Magdalena y Bahia Almejas condiciones favorables para $C$. gigas en cuanto al ambiente general por lo menos durante los meses de invierno y primavera. Una dificultad aparente es que en esta zona existen numerosos predadores y quizá parásitos del ostión de mangle, pero pensamos que mediante las técnicas de suspensión se evitarán estas dificultades, principalmente porque se va a suspender el ostión de balsas, evitando contacto con el fondo. En el presente no es posible decir si llevarán estos experimentos a resultados positivos, pero si se logra el mismo resultado que en Bahía San Quintín será razonable suponer que se podrá con el tiempo y la inversión adecuada, crear una cosecha que ascenderá en valor a las coosechas probables mencionadas para el pescado.

Hasta ahora se ha tratado el pescado y el cultivo como fuentes de ingreso económico. También se deberán de considerar como fuentes de ingresos de proteínas. Si se puede efectuar una cosecha anual de aproximadamente 1,600 Tm de carne de pescado, y si asumimos que de esos 1,600 Tm aproximadamente $400 \mathrm{Tm}$ sean de carne sin hueso, y si asumimos también que una persona que come 250 
9 de carne de pescado ha comido satisfactoriamente en cuanto a carne se refiere, se puede calcular cuántas comidas de pescado se obtendrán:

$$
\frac{400,000}{0.25}=1,600,000
$$

Entonces 1,600,000 comidas de proteínas satisfactorias serían cosechadas nualmente del área. Asumiendo que la población del Estado de Baja California Sur tiene una población de 80,000 personas, esto equivale a decir que cada uno de ellos comería bien 20 veces por año. Alternativamente asumiendo que viven 5,000 personas en la vecindad de Bahía Magdalena, estas personas podrían comer 320 días al año de la carne de pescado de la Bahía. El hecho de que aquí tratamos de una fuente de carne barata, que resulta negativa desde el punto de vista comercial, resulta positivo desde el punto de vista social.

Esto nos lleva inmediatamente a la consideración de una serie de problemas que quedan muy afuera de la órbita del biólogo pesquero, pero que el científico pesquero tiene deber de indicar, ya sea para dar ideas precisas por lo menos para indicar pistas que se deban de seguir, y que otros científicos pesqueros con otras especialidades, podrán y deberón seguir.

Uno de los problemas para la pesca en el Estado de Baja California Sur es la falta de un mercado constante. Esto se debe a una serie de factores: Los precios bajos al pescador, impidiendo la pesca, mientras que el precio frecuentemente alto al consumidor impide el consumo. La falta de higiene en los campos pesqueros, para quien los haya visitado, es horrorizante y lleva directamente al mercado de productos inferiores que en su turno, disminuyen la demanda. La falta de medios de transporte adecuados incrementa el problema de higiene, problema de todos modos agravado por las dificultades que son consecuencias del acceso difícil al agua que existe, por lo menos en la zona de Bahía Magdalena. La falta de fuentes de hielo en ciertas zonas puede ser crítica, y esto es particularmente aplicable a la zono de Bahía Magdalena y Bahía Amajas. la falta de medios de transportacion $\mathrm{sa}$. nitarios y de manejo higiénico del pescado en los campos pesqueros, levcin o un producto menos bueno y a be mer cado más diffeil La falta de rquipos adecuados y embarcaciones adecudas también resulia ser problema. Todos estos aspectos técnicos y económicos deben ser considerados en todos sus iotclies. Hasta ahora se ha tratado la zona de Bahía Magdalena como fuente de dinero y por consecuencia, de emplecs, $y$ como fuente de proteinas. Se deba tam. bién considerar como zona de refugio y zona de cría.

Es la zona de estudio una zona de cría para muchas especies de importancia comercial actual y potencial. Por ejemplo, para Micropogon ectenes y Menticirrus undulatus, es una zona donde, en agosto, se encontraron numercsicinios. pescaditos de $5-10 \mathrm{~cm}$ que claromente son los iviveniles resultados del cesove precederite. Las ballenas grises usan el area como zona de refugio para los balienatos durante sus migraciones. El lenguado, Paralichthys californicus, que llega a su punto de distribución sureño en Bahía Magdalena, puede también usar esta zona para cría.

En cuanto al camarón se refiere, parece abundar más el azul en Bahía Almejas y el café en Bahía Magdalena.

Todos estos problemas se subrayan por el hecho del desarrollo de Puerto San Carlos, al norte de Bahía Magdalena, como puerto de altura para barcos de hasta 15,000 toneladas de desplazamiento. El desarrollo de este puerto tenderá a introducir contaminantes importantes a la Bahia, y es probable que éstos sean más nocivos para los peces pequeños que los grandes. Al mismo tiempo los peces grandes usados como fuente de proteína para el hómbre, podrán acumular los contaminantes a niveles relativamente altos. Afortunadainente las zonas principales de cría parecen estar alrededor de Bahía Almejas, y quizás serán protegidas en alguna medida de la contaminación. Al mismo tiempo, esto indica la necesidad de un estudio detallado de las corrientes diurnas y estacionaies en las bahías. 


\section{P. MATHEWS}

Las fuentes de contaminantes tendrán también importantísima influencia en la cosecha del ostión de mangle $y$ en el cultivo de ostión: los ostiones son de las especies que más acumulan contaminantes como los metales pesados, los organoclorados y el petróleo. La ingestión de ostiones contaminados puede resultar en una importante fuente de contaminación para el hombre. El desarrollo del puerto producirá un incremento en todos estos importancia primordial la realización de un estudio de los niveles actuales de contaminantes para tener un punto base en nuestros conocimientos: sin tal punto base nos será imposible controlar los contaminantes industriales puesto que los contaminadores argumentarán, no sin éxito, que "todo eso venía desde antes".

Hasta cierto nivel, el desarrollo de San Carlos como puerto podría resultar inconsistente can el mejor aprovechamiento para la zona en cuanto al cultivo de ostión. Se deben de considerar de las dos alternativas los beneficios al hombre, y el punto hacia el cual éstas actividades se pueden armonizar. Un método de intentar una armonización sería la ubicación del cultivo en zonas como Bahía Almejas que resultarían más distanciadas de las fuentes de contaminación.

Hasta ahora se ha tratado únicamente dè las poblaciones demersales y de ostión de mangle. Hay que notar la presencia de otras poblaciones de moluscos comerciales hasta ahora muy poco conocidos en cuanto a distribución y abundancia, y también las poblaciones muy importantes de pescados pelágicos. De la sardina se cosechan $15,000 \mathrm{Tm}$ anualmente esta pesquería está basada en Puerto Adolfo López Mateos, situado en los canales a 11 millas al norte de Bahía Magdalena. La sardina pescada es de dos especies, la crinuda y la monterrey. El área de Bahía Magdalena es pequeña para una cosecha tan grande $y$ de todos modos los datos enseñan que las poblaciones de sardina entran y salen a la Bahía de Almejas. Los estudios del grupo de ictioplancton de la Unidad dee Ciencias Marinas indican que la anchoveta de la cual tanto se habla como fuente de ingresos futuros, desova en toda la zona de Bahía Magdalena.

La industrialización del área, con la contaminación consiguiente, puede tener efectos importantes en las poblaciones pelágicas que tenderán a llevar a la contaminación del hombre mismo.

\section{AGRADECIMIENTOS}

Quiero agradecer la cooperación de los pescadores de la Cooperativa "Isla Macapule" de Guasave, Sin. y al Capitán Miguel Evaristo Cárdenas del barco Macapule I. También agradecemos la cooperación de la Secretaría de Recursos Hidráulicos con quien se efectuaron estos trabajos bajo cantrato con la Dirección de Acuacultura. También agradecemos la ayuda que nos prestó el P.O. Javier Amador Buenrostro Residente de la Dirección de Aculcultura en La Paz, B. C. Sur, y al ing. Sonín Pérez Pérez Director de la Escuela Tecnológica Pesquera de San Carlos, B. C. Sur y al Ocean. José Pérez Higuera Sub-Director de la misma. También queremos reconocer la cooperación y ayuda del Biol. Manuel Flores Villegas Jefe de Pesca en Baja California Sur; al comandante del Sector Naval de Puerto Cortés, B. C. Sur Vicealmirante Argudín, y a todos los otros oficiales del Gobierno Federal que nos facilitaron los trabajos en esas zonas. 